\title{
Formaldehyde biodegradation and its inhibitory effect on nitrification
}

\author{
Marta Eiroa, Christian Kennes, María C Veiga \\ Journal of Chemical Technology \& Biotechnology, Volume 79, Issue 5, May 2004, \\ Pages 499-504 \\ DOI: $10.1002 / j c t b .1011$
}

\begin{abstract}
The simultaneous removal of formaldehyde and ammonium in aerobic cultures and the inhibitory effect of formaldehyde on ammonium oxidation were investigated. The influence of a co-substrate, methanol, on formaldehyde biodegradation and on the nitrification process was also evaluated. Formaldehyde was completely removed at all concentrations tested (30-3890 $\mathrm{mg} \mathrm{dm}-3$ ) in assays with that compound as the single carbon source and in the presence of methanol as co-substrate. An initial formaldehyde biodegradation rate of $4.6 \mathrm{~g} \mathrm{CH} 2 \mathrm{O}$ g-1 VSS d-1 was obtained for $2000 \mathrm{mg} \mathrm{CH} 2 \mathrm{O}$ $\mathrm{dm}-3$ as single carbon source compared with a rate of $7.3 \mathrm{~g} \mathrm{CH} 2 \mathrm{O} \mathrm{g}-1 \mathrm{VSS} d-1$ when methanol was added. Formaldehyde was inhibitory to the nitrification process at initial concentrations higher than $350 \mathrm{mg} \mathrm{dm}-3$. Increasing the initial formaldehyde concentration or adding a co-substrate such as methanol resulted in a longer lag phase before ammonium oxidation and caused a decrease in the degree of nitrification. Nitrification was completely inhibited at initial formaldehyde concentrations higher than $1500 \mathrm{mg} \mathrm{dm}-3$.
\end{abstract}

\section{Keywords:}

Formaldehyde; methanol; ammonium; toxicity; nitrification 


\section{INTRODUCTION}

Wastewaters from synthetic resin-producing industries are characterized by the presence of high levels of nitrogen compounds (56-1462 mg TKN dm-3), mainly ammonium, and organic matter including formaldehyde as the major component (7-2711 mg CH2O $\mathrm{dm}-3) .1$ The activated sludge process is one of the most common biological treatments of such wastewaters, in which ammonium is removed by bacteria in two successive steps. During the nitrification step, ammonium is oxidized to nitrate under aerobic conditions; and during the denitrification step, nitrate is reduced to molecular nitrogen in the presence of a carbon source under anoxic conditions. The nitrification process itself is also carried out in two successive stages, namely the conversion of ammonium to nitrite by ammonium oxidizers followed by the further conversion of nitrite to nitrate by nitrite oxidizers. Both groups of bacteria are autotrophic, utilizing carbon dioxide as the carbon source for biosynthesis, and obtaining energy from the oxidation of nitrogen. Nitrification is commonly the rate limiting step of the overall nitrogen removal kinetics.

Formaldehyde, which is produced through the catalytic oxidation of methanol, is a common compound in the chemical industry, used in a wide variety of processes and frequently found in wastewaters and waste gases. 2 Because of its toxicity, formaldehyde is often used as an active ingredient in preservatives and disinfectant agents to inhibit microbial activity. Therefore, its presence is expected to inhibit biological nitrogen removal in aerobic wastewater treatment plants.

A few studies have shown that formaldehyde can inhibit the growth of aerobic bacteria at very low concentrations.3, 4 In spite of its inhibitory effect to microorganisms, formaldehyde is known to be biodegradable under aerobic conditions. Bonastre et al5 reported the partial biodegradation of formaldehyde at concentrations of $2300 \mathrm{mg} \mathrm{dm}-3$ in tests with activated sludge. In another study, it was reported that Pseudomonas putida A2 degraded $400 \mathrm{mg} \mathrm{dm}-3$ formaldehyde as the sole carbon source in batch assays. 3 The degradation was initiated by a dismutation reaction, yielding formic acid and methanol as products. The biodegradation of these metabolites started after exhaustion of formaldehyde in the medium. Gerike and Gode4 observed that $30 \mathrm{mg} \mathrm{dm}-3$ formaldehyde inhibited oxygen consumption in activated sludge whereas Zagornaya et al6 reported the complete biodegradation of $2300 \mathrm{mg} \mathrm{dm}-3$ formaldehyde and $2400 \mathrm{mg}$ $\mathrm{dm}-3$ phenol in wastewater treated in an activated sludge plant. More recently, it was found that Halomonas sp MA-C was able to degrade $100 \mathrm{mg} \mathrm{dm}-3$ formaldehyde in a salt medium.7 Yamazaki et al8 studied the biodegradation of formaldehyde by a formaldehyde-resistant bacterium isolated from coastal seawater and found that this bacterium could degrade up to $400 \mathrm{mg} \mathrm{dm}-3$ formaldehyde in a medium containing 3\% sodium chloride. Hidalgo et al9 investigated formaldehyde biodegradation by Rhodococcus erythropolis UPV-1 in the presence of phenol. Formaldehyde was removed both as the single carbon source as well as in the presence of $800 \mathrm{mg} \mathrm{dm}-3$ phenol. Hardly any data have been published on the simultaneous removal of nitrogen compounds and formaldehyde as single carbon source or in the presence of a cosubstrate. 
The purpose of this research was to study the simultaneous removal of formaldehyde and ammonium in activated sludge cultures. The biodegradability of formaldehyde and its toxic effect on the nitrification process were investigated in batch assays. The effect of methanol on formaldehyde biodegradation and on the nitrification process was evaluated as well. First, assays with formaldehyde as single carbon source and, then, assays with formaldehyde and methanol were undertaken for comparison. Such information is useful for optimizing the treatment of wastewaters containing nitrogen compounds and formaldehyde, and in which other organic compounds such as methanol may also appear, as in synthetic resin-producing industries.

NOTATION

\begin{tabular}{|ll|}
\hline COD & Chemical oxygen demand \\
\hline TKN & Total Kjeldahl nitrogen \\
\hline VSS & Volatile suspended solids \\
\hline
\end{tabular}

\section{MATERIALS AND METHODS}

\section{Analytical methods}

Formaldehyde was analysed spectrophotometrically according to the Hantzch reaction.10 A properly diluted formaldehyde solution was supplemented with acetyl acetone and ammonium acetate to form a coloured compound. The concentration of this compound was measured at $412 \mathrm{~nm}$ using a Perkin Elmer Lambda $11 \mathrm{UV} / \mathrm{Vis}$ spectrophotometer.

Nitrite and nitrate anions were analysed by capillary electrophoresis using a Hewlett Packard 3DCE system with a micro-capillary tube of fused silica $(40 \mathrm{~cm} \times 50 \mu \mathrm{m})$. Determinations were performed with the detector end of the column at $30 \mathrm{kV}$ and at a column temperature of $40{ }^{\circ} \mathrm{C}$. A sodium phosphate solution was employed as the electrolyte, and UV detection was undertaken at a wavelength of $214 \mathrm{~nm}$ and $450 \mathrm{~nm}$ as reference.

Ammonium, $\mathrm{pH}$, total suspended solids and volatile suspended solids were evaluated according to the method described in Ref11.

\section{Batch assays}

After defining the most appropriate operating conditions in scouting experiments, nitrifying batch assays were undertaken in $500 \mathrm{~cm} 3$ vials filled with $100 \mathrm{~cm} 3$ medium. Each flask was inoculated with $3.5 \mathrm{~g}$ VSS dm-3, using a sludge obtained from the aerobic chamber of the full-scale wastewater treatment plant of a synthetic resinproducing factory. 1 The initial $\mathrm{pH}$ was adjusted to 7.5 using NaHCO3 buffer. The 
medium was supplemented with $10 \mathrm{~cm} 3$ nutrient solution composed of ( $\mathrm{g} \mathrm{dm}-3)$ : MgSO4.7H2O, 0.12; KH2PO4, 0.25; (NH4)2SO4, 1.18; NaCl, 1.00 and NH4Cl, 0.96; and with $0.05 \mathrm{~cm} 3$ trace elements solution containing ( $\mathrm{g} \mathrm{dm}-3)$ : $\mathrm{CaCl} 2.2 \mathrm{H} 2 \mathrm{O}, 7.35$; $\mathrm{MnCl}$.4H2O, 5.06; FeSO4.7H2O, 5.00; (NH4)6Mo7O24.4H2O, 1.05; Na2(EDTA), 50.00; CuSO4.5H2O, 1.57; CoCl2.6H2O, 1.57 and ZnCl2, 1.04. Finally, 250 mg NH4+$\mathrm{N} \mathrm{dm}-3$ and formaldehyde either without or with methanol (12.5\%) were added in order to study the nitrification process and formaldehyde biodegradation. Formaldehyde concentrations ranging from 30 to $3890 \mathrm{mg} \mathrm{dm}-3$ were assayed. Formaldehyde, nitrite and nitrate concentrations were measured at regular time intervals.

Assays were performed in a thermostatic chamber at $20^{\circ} \mathrm{C}$ and with constant shaking at $200 \mathrm{rpm}$. All experiments were carried out in duplicate. Control assays without biomass and others with sterilized biomass were also prepared in order to quantify the extent of formaldehyde removal through abiotic processes such as volatilization, adsorption and chemical transformation. Control assays with sterilized biomass were autoclaved at 120 ${ }^{\circ} \mathrm{C}$ for $20 \mathrm{~min}$.

\section{RESULTS AND DISCUSSION}

\section{Biodegradation of formaldehyde as the single carbon source}

The formaldehyde concentration remained constant in uninoculated control vials during all experiments, demonstrating that there was no abiotic removal of formaldehyde. In the vials inoculated with sterilized biomass a discrepancy was observed between the added formaldehyde concentration and the initial concentration measured at time zero. Afterwards, the formaldehyde concentration remained constant. Therefore, formaldehyde removal caused by initial adsorption to the biomass did take place, the amount adsorbed being around 23\%. Losses of formaldehyde by bioadsorption and chemical transformation have been reported by other investigators.12, 13 In the biodegradation assays with active biomass, this discrepancy between the added formaldehyde concentration and the initial experimental concentration was also observed; the amount adsorbed being always around 38\%. Therefore, the concentrations plotted in all the figures are those measured analytically by the Hantzch reaction.

Formaldehyde biodegradation was investigated at concentrations ranging from 30 to $3890 \mathrm{mg} \mathrm{dm}-3$ in batch assays (Fig 1). The data show that high formaldehyde concentrations of up to $3890 \mathrm{mg} \mathrm{dm}-3$ could be removed using that pollutant as the single carbon source. Complete formaldehyde biodegradation took place in less than 24 h. The initial substrate consumption rates (rs) versus initial formaldehyde concentrations are shown in Fig 2. The results show the relatively fast and complete removal of high initial formaldehyde concentrations. The increase in initial formaldehyde concentration caused a steady increase of the initial formaldehyde degradation rate, up to the highest concentration of $3890 \mathrm{mg} \mathrm{dm}-3$, suggesting first order kinetics $(\mathrm{R} 2=0.9872)$ and no inhibition effect. Initial substrate consumption rates between 0.22 and 8.2 g CH2O g-1 VSS d-1 were obtained for formaldehyde concentrations ranging from 30 to $3890 \mathrm{mg}$ $\mathrm{dm}-3$. The first order rate constant was $0.31 \mathrm{~h}-1$. 

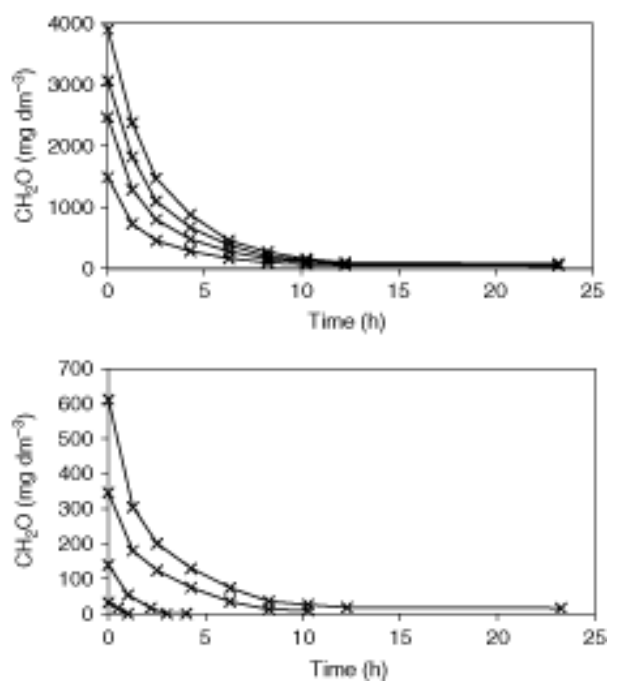

Figure 1.

Formaldehyde biodegradation at different initial concentrations versus time.

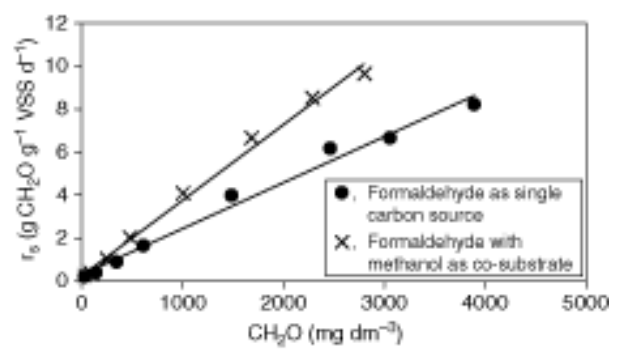

Figure 2.

Initial substrate consumption rates (rs) versus initial formaldehyde concentrations.

The sludge used in this study tolerated much higher formaldehyde concentrations than reported in the literature. Bonastre et al5 reported the partial biodegradation of formaldehyde at concentrations of $2300 \mathrm{mg} \mathrm{dm}-3$ in aerobic assays with activated sludge. They observed that the experimental data did not follow first order kinetics and tended to higher orders. The much higher concentrations removed in our assays could have resulted from the long-term (several years) adaptation of the sludge to formaldehyde in the industrial wastewater treatment plant from which the present inoculum was obtained.

\section{Biodegradation of formaldehyde with methanol as co-substrate}

Formaldehyde biodegradation at similar concentrations to those used in the previous study was investigated in the presence of methanol (12.5\%) (Fig 3). The influence of the co-substrate methanol on formaldehyde removal is of interest because the alcohol is used in the industrial production of formaldehyde. Methanol has also been detected in the full-scale wastewater treatment plant operating at the synthetic resin-producing factory (unpublished data). Sometimes it is also added on purpose to reach the optimal COD/TKN ratio for the denitrification process. 

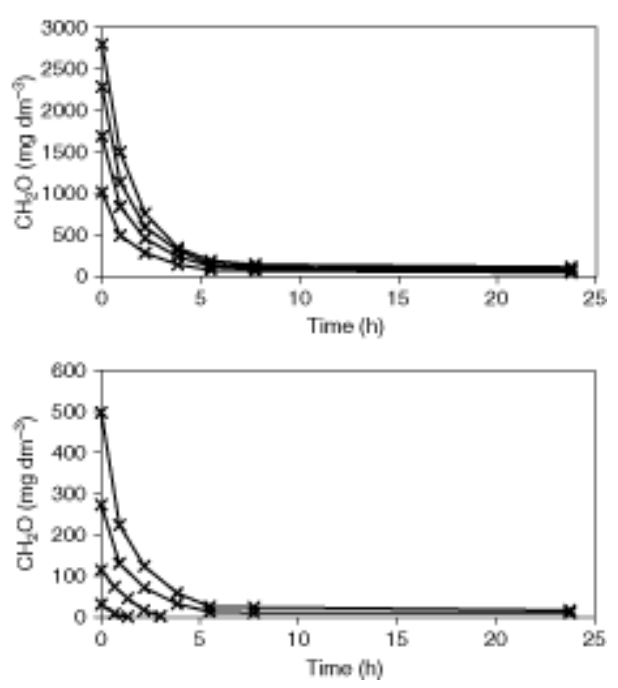

Figure 3.

Formaldehyde biodegradation at different concentrations versus time in the presence of methanol.

The formaldehyde concentration remained constant during all experiments in uninoculated control vials. There was no abiotic formaldehyde removal, as has already been reported in the case of assays with formaldehyde as the single carbon source. In the vials inoculated with sterilized biomass a discrepancy was observed between the added formaldehyde concentration and the measured initial concentration, suggesting formaldehyde removal caused by adsorption to the biomass. The methanol concentration remained constant during all experiments in uninoculated control vials and in vials inoculated with sterilized biomass. Therefore, methanol removal caused by abiotic processes or initial adsorption to the biomass did not take place.

In the presence of methanol, the amount of formaldehyde adsorbed was around 55\%, being higher than with formaldehyde alone. As observed in the previous assays, without co-substrate, formaldehyde was again completely biodegraded in all cases and biodegradation took place in less than $24 \mathrm{~h}$ (Fig 3). The initial substrate consumption rates (rs) versus initial formaldehyde concentrations are shown in Fig 2. The increase in initial formaldehyde concentration caused a steady increase of the initial formaldehyde degradation rate, suggesting first order kinetics $(\mathrm{R} 2=0.9936)$ and no inhibition effect. Higher formaldehyde biodegradation rates were obtained with methanol as co-substrate rather than without it (Fig 2). For instance, a rate of 4.6 g CH2O g-1 VSS d-1 was obtained for $2000 \mathrm{mg} \mathrm{CH} 2 \mathrm{O} \mathrm{dm}-3$ as single carbon source compared with a rate of 7.3 g CH2O g- 1 VSS $d-1$ when $12.5 \%$ methanol was added. The first order rate constant was $0.51 \mathrm{~h}-1$.

In the literature, data for formaldehyde biodegradation in the presence of a co-substrate have not been published in the case of aerobic cultures. In anaerobic cultures, Omil et al14 reported that initial formaldehyde biodegradation rates with that pollutant as the sole carbon source $(0.027-0.042$ g CH2O g-1 VSS d-1) were lower than in presence of 
volatile fatty acids as co-substrate $(0.051-0.081 \mathrm{~g}$ CH2O g-1 VSS d-1). The possibility of formaldehyde biodegradation despite the presence of an alternative readily metabolizable carbon source is a characteristic of significant practical interest when formaldehyde needs to be removed in environments containing other carbon sources, as in the case of wastewaters from synthetic resin-producing factories.

\section{Nitrification in the presence of formaldehyde}

Nitrification was investigated both without formaldehyde and in the presence of different formaldehyde concentrations. In nitrification assays the $\mathrm{pH}$ was between 7.5 and 8.2, which is close to the optimum value of around 7.8 for nitrifiers.15 Nitrification was possible with limited inhibition at low initial formaldehyde concentrations. Inhibitory effects increased at higher initial formaldehyde concentrations in batch assays, contrary to results obtained in a continuous reactor in which no inhibition was observed for formaldehyde concentrations of $3168 \mathrm{mg} \mathrm{dm}-3$ (unpublished data). Some representative curves are presented in Fig 4. Nitrite production was observed in a first stage; later nitrite was transformed into nitrate. In assays without formaldehyde and with $30 \mathrm{mg} \mathrm{dm}-3$ formaldehyde the lag phases before nitrite and then nitrate production were similar. In assays with higher initial formaldehyde concentrations, the lag phase was longer. In addition, at initial formaldehyde concentrations above $350 \mathrm{mg} \mathrm{dm}-3$ the level of nitrification started to decrease. At concentrations above $1500 \mathrm{mg} \mathrm{dm}-3$, the amounts of both nitrite and nitrate were below detection limits, showing that the nitrification process was completely inhibited. Thus, the increase in initial formaldehyde concentration caused a longer lag phase before the nitrification process started as well as a decrease in the nitrification percentage with accumulation of ammonium (Fig 4).
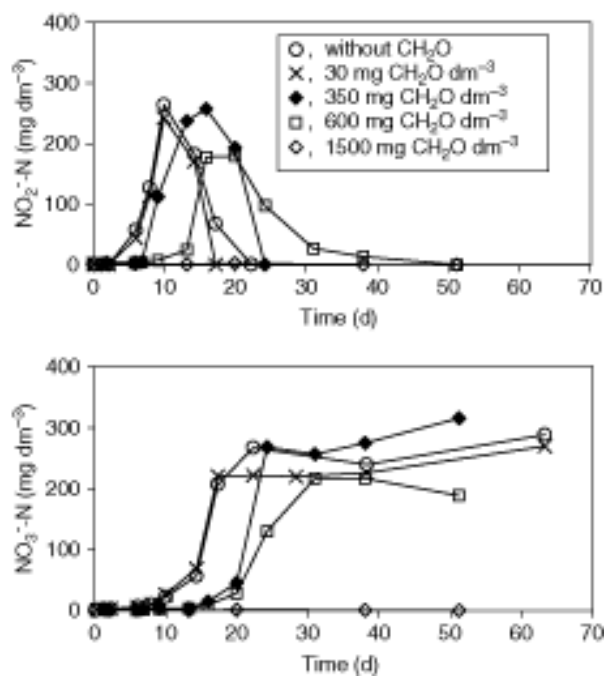

Figure 4. Ammonium nitrification in the presence of different formaldehyde concentrations.

Gómez et al16 studied the effect of different concentrations of several volatile organic compounds on the nitrification process in batch cultures. They also found that an increase in the concentration of the volatile organic compound provoked an increase in the degree of inhibition of the nitrification process. However, nitrite was not detected at 
any concentration of volatile organic compounds, unlike our assays. In our case, low dissolved oxygen concentrations were present at the end of the experiments although the value never dropped below $1 \mathrm{mg} \mathrm{dm}-3$. Oxygen concentrations did not affect ammonium oxidation but reduced nitrite oxidation, leading to an accumulation of nitrite and delaying nitrate formation. Hanaki et al17 have also reported that low dissolved oxygen concentrations did not adversely affect ammonium oxidation. They observed that the growth yield of ammonium oxidizers was higher at low dissolved oxygen levels, and this positive effect compensated for the reduced ammonium oxidation rate per unit biomass. Nitrite oxidation was strongly inhibited by low dissolved oxygen concentrations and the growth yield of nitrite oxidizers remained unchanged.

In our own study, formaldehyde and ammonium were both added in all the assays and a simultaneous growth of heterotrophic biomass and nitrifiers took place, leading to very low nitrification rates in comparison with the biodegradation rates of formaldehyde. The inhibitory effect on the nitrification process could be explained by the high COD/TKN ratio which was favourable to the fast growing heterotrophic bacteria delaying the development of the nitrifying population. As was reported by Cheng and Chen,18 high organic matter concentrations stimulate the growth of heterotrophic bacteria, which compete with the nitrifiers for their basic substrates, ammonium and oxygen.

\section{Nitrification in the presence of formaldehyde and methanol}

Nitrification was investigated in the presence of similar formaldehyde concentrations as in the previous study but with $12.5 \%$ methanol (Fig 5). Similar behaviour to assays of nitrification in the presence of formaldehyde alone was observed. The difference was that the nitrification process started slightly later, meaning that the adaptation period needed by the microorganisms was longer. Further, the inhibitory effect on nitrification was greater at lower formaldehyde concentrations. In the presence of methanol, at initial formaldehyde concentrations above $175 \mathrm{mg} \mathrm{dm}-3$, the level of nitrification started to decrease. At initial concentrations above $500 \mathrm{mg} \mathrm{dm}-3$, both nitrite and nitrate concentrations were near zero, showing that the nitrification process was completely inhibited. This difference could be explained by the fact that the COD/TKN ratio was higher in the assays with formaldehyde and methanol as carbon sources than in the assays without methanol. Therefore, the competition between heterotrophic bacteria and nitrifiers was higher. 

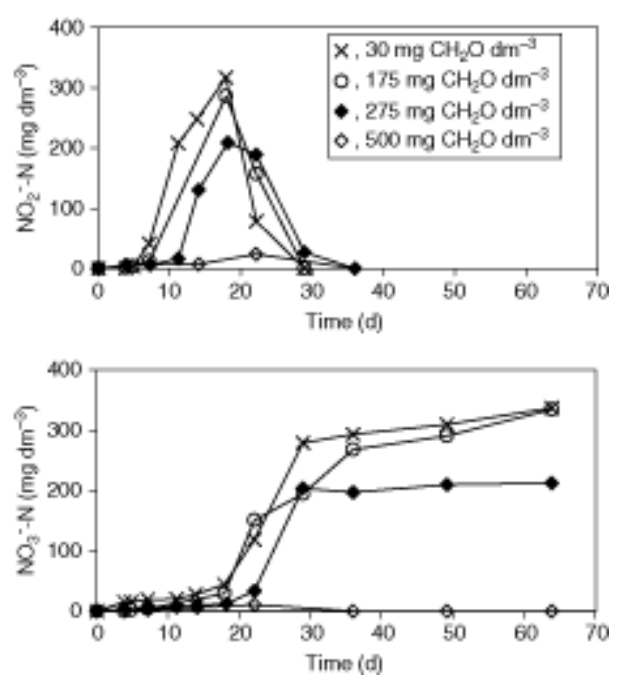

Figure 5.

Ammonium nitrification in presence of different formaldehyde concentrations and $12.5 \%$ methanol.

\section{CONCLUSIONS}

Formaldehyde as the single carbon source was degraded at concentrations ranging from 30 to $3890 \mathrm{mg} \mathrm{dm}-3$. The initial biodegradation rates increased in the presence of increasing initial concentrations of that compound, reaching 8.2 g CH2O g-1 VSS d-1 for $3890 \mathrm{mg} \mathrm{CH} 2 \mathrm{O} \mathrm{dm}-3$.

Formaldehyde was more rapidly and also completely removed at similar concentrations in the presence of $12.5 \%$ methanol as co-substrate. For example, an initial formaldehyde biodegradation rate of 4.6 g CH2O g-1 VSS d-1 was obtained for $2000 \mathrm{mg}$ CH2O $\mathrm{dm}-3$ as single carbon source compared with a rate of $7.3 \mathrm{~g} \mathrm{CH} 2 \mathrm{O}$ g- 1 VSS d-1 when $12.5 \%$ methanol was added.

Formaldehyde inhibited the nitrification process, showing higher inhibition levels with increasing initial formaldehyde concentration. Nitrification was completely inhibited at initial formaldehyde concentrations higher than $1500 \mathrm{mg} \mathrm{dm}-3$.

When methanol was added as co-substrate, the nitrification process was inhibited at lower formaldehyde concentrations, being completely inhibited at initial formaldehyde concentrations above $500 \mathrm{mg} \mathrm{dm}-3$.

\section{Acknowledgements}

The present research was financed by the Spanish Ministry of Science and Technology (Projects REN2000-0218-P4-02 and PPQ2002-02882). M Eiroa is grateful to the Spanish Ministry of Education and Culture for a $\mathrm{PhD}$ fellowship. 


\section{REFERENCES}

Cantó M, Gómez J, Kennes C and Veiga MC, Integrated anoxic-aerobic treatment of wastewaters from a synthetic resin producing factory, in European Conference on New Advances in Biological Nitrogen and Phosphorus Removal for Municipal or Industrial Wastewaters, Narbonne, France, pp 289-292 (1998).

2Prado ÓJ, Eiroa M, Veiga MC and Kennes C, Bioreactors for the treatment of industrial waste gases containing formaldehyde and other aliphatic compounds, in Focus on Biotechnology, ed by AgathosSN and ReinekeW. Kluwer Academic Publishers, Dordrecht, The Netherlands Vol 3C, pp 259-273 (2003).

3Adroer N, Casas C, de Mas C and Solà C, Mechanism of formaldehyde biodegradation by Pseudomonas putida. Appl Microbiol Biotechnol 33: 217-220 (1990).

$\square$ 4Gerike K and Gode P, The biodegradability and inhibitory threshold concentration of some disinfectants. Chemosphere 21: 799-812 (1990).

5Bonastre $\mathrm{N}$, de Mas $\mathrm{C}$ and Solà $\mathrm{C}$, Vavilin equation in kinetic modelling of formaldehyde biodegradation. Biotechnol Bioeng 28: 616-619 (1986).

6Zagornaya PB, Denis AD, Gvozdyak PI, Nikonenko VU and Chehovskaya TP, Microbiological purification of above resin wastewaters. Biotekhnologiya 2: 51-53 (1990).

7Azachi M, Henis Y, Oren A, Gurevich P and Sarig S, Transformation of formaldehyde by a Halomonas sp. Can J Microbiol 41: 548-553 (1995).

8Yamazaki T, Tsugawa $\mathrm{W}$ and Sode $\mathrm{K}$, Biodegradation of formaldehyde by a formaldehyde-resistant bacterium isolated from seawater. Appl Biochem Biotechnol 9193: 213-217 (2001).

9Hidalgo A, Lopategi A, Prieto M, Serra JL and Llama MJ, Formaldehyde removal in synthetic and industrial wastewater by Rhodococcus erythropolis UPV-1. Appl Microbiol Biotechnol 58: 260-263 (2002).

10Nash T, The colorimetric estimation of formaldehyde by means of the Hantzch reaction. Biochem J 55: 416-421 (1953).

11APHA, American Public Health Association Ed ClesceriLS, BreenbergAE and EatonAD. Standard Methods for the Examination of Water and Wastewater, 20th edn. Washington, DC, USA (1998).

12Bhattacharya SK and Parkin GF, Fate and effect of methylene chloride and formaldehyde in methane fermentation systems. J Water Pollut Control Fed 60: 531536 (1988). 
13González-Gil G, Kleerebezem R, van Aelst A, Zoutberg GR, Versprille AI and Lettinga $G$, Toxicity effects of formaldehyde on methanol degrading sludge and its anaerobic conversion in biobed ${ }^{\circledR}$ expanded granular sludge bed (EGSB) reactors. Water Sci Technol 40: 195-202 (1999).

14Omil F, Méndez D, Vidal G, Méndez R and Lema JM, Biodegradation of formaldehyde under anaerobic conditions. Enz Microbiol Technol 24: 255-262 (1999).

15Antoniou P, Hamilton J, Koopman B, Jain R, Holloway B, Lyberatos G and Svoronos SA, Effect of temperature and $\mathrm{pH}$ on the effective maximum specific growth rate of nitrifying bacteria. Water Res 24: 97-101 (1990).

16Gómez J, Méndez R and Lema JM, Kinetic study of addition of volatile organic compounds to a nitrifying sludge. Appl Biochem Biotechnol 87: 189-202 (2000).: 6

17Hanaki K, Wantawin C and Ohgaki S, Nitrification at low levels of dissolved oxygen with and without organic loading in a suspended-growth reactor. Water Res 24: 297-302 (1990).

18Cheng S and Chen W, Organic carbon supplement influencing performance of biological nitritification in a fluidized bed reactor. Water Sci Technol 30: 131-142 (1994). 